\title{
Polyubiquitination of the demethylase Jhd 2 controls histone methylation and gene expression
}

\author{
Douglas P. Mersman, Hai-Ning Du, Ian M. Fingerman, Paul F. South, and Scott D. Briggs ${ }^{\mathbf{1}}$ \\ Department of Biochemistry, Purdue University, West Lafayette, Indiana 47907, USA; and the Purdue Cancer Center, Purdue \\ University, West Lafayette, Indiana 47907, USA
}

\begin{abstract}
The identification of histone methyltransferases and demethylases has uncovered a dynamic methylation system needed to modulate appropriate levels of gene expression. Gene expression levels of various histone demethylases, such as the JARID1 family, show distinct patterns of embryonic and adult expression and respond to different environmental cues, suggesting that histone demethylase protein levels must be tightly regulated for proper development. In our study, we show that the protein level of the yeast histone H3 Lys 4 (H3 K4) demethylase Jhd2/ $\mathrm{Kdm} 5$ is modulated through polyubiquitination by the E3 ubiquitin ligase Not4 and turnover by the proteasome. We determine that polyubiquitin-mediated degradation of Jhd2 controls in vivo $\mathrm{H} 3 \mathrm{~K} 4$ trimethylation and gene expression levels. Finally, we show that human NOT4 can polyubiquitinate human JARID1C/SMCX, a homolog of Jhd2, suggesting that this is likely a conserved mechanism. We propose that Not4 is an E3 ubiquitin ligase that monitors and controls a precise amount of Jhd2 protein so that the proper balance between histone demethylase and histone methyltransferase activities occur in the cell, ensuring appropriate levels of $\mathrm{H3} \mathrm{K} 4$ trimethylation and gene expression.
\end{abstract}

[Keywords: Jhd2; chromatin; histone methylation; demethylase; ubiquitination; proteasome]

Supplemental material is available at http://www.genesdev.org.

Received December 4, 2008; revised version accepted March 12, 2009.

The identification of both histone methyltransferases and histone demethylases implies that a dynamic methylation system exists in eukaryotes. This dynamic methylation system is needed to modulate appropriate levels of gene expression via histone lysine methylation and methyl-binding factors. In Saccharomyces cerevisiae, histone H3 K4, K36, and K79 methylation has been associated with gene activation, and dynamic methylation changes have been detected for histone H3 K4 and H3 K36 methylation (Ng et al. 2003; Ingvarsdottir et al. 2007; Klose et al. 2007; Liang et al. 2007; Seward et al. 2007; Tu et al. 2007).

Set1 is the sole SET domain-containing enzyme that is necessary for catalyzing histone H3 K4 mono-, di-, and trimethylation in S. cerevisiae (Briggs et al. 2001; Roguev et al. 2001). It is required for silencing at the rDNA, telomeres, and mating-type loci and has been purified as a multiprotein complex (Nislow et al. 1997; Briggs et al. 2001; Miller et al. 2001; Roguev et al. 2001; Bryk et al. 2002; Fingerman et al. 2005). In addition, Set1 and H3 K4 trimethylation are enriched at the $5^{\prime}$ ends of the open

${ }^{1}$ Corresponding author.

E-MAIL sdbriggs@purdue.edu; FAX (765) 494-7897.

Article published online ahead of print. Article and publication date are online at http://www.genesdev.org/cgi/doi/10.1101/gad.1769209. reading frames of transcriptionally active genes in both yeast and higher eukaryotes (Santos-Rosa et al. 2002; Ng et al. 2003; Schneider et al. 2004). These and other studies suggest that Set1 and H3 K4 trimethylation play positive roles in regulating gene expression (Dehe and Geli 2006).

The JmjC domain-containing protein Jhd2 was recently purified from budding yeast as a monomeric subunit and was shown to have in vitro histone $\mathrm{H} 3 \mathrm{~K} 4$ demethylase activity (Liang et al. 2007). Jhd2 was also shown to remove $\mathrm{H} 3 \mathrm{~K} 4 \mathrm{di}$ - and trimethylation in vivo after Set1 was depleted from the cells (Seward et al. 2007). However, overexpressing Jhd 2 in cells expressing Set 1 showed only slight but detectable decreases in $\mathrm{H} 3 \mathrm{~K} 4$ trimethylation (Liang et al. 2007). Deletion of Jhd2 has been shown to have only a minor effect on GAL1 gene expression and results in a less than twofold increase in H3 K4 trimethylation levels at the $5^{\prime}$ end of the GAL1 gene (Ingvarsdottir et al. 2007).

In addition to histone methyltransferases and demethylases, ubiquitin conjugating enzymes and ubiquitin ligases are important for maintaining appropriate levels of H3 K4 methylation. Rad6 and Bre1 are E2 ubiquitin conjugating and E3 ubiquitin ligase enzymes, respectively, that have been shown to be necessary for $\mathrm{H} 2 \mathrm{~B}$ K123 monoubiquitination (Robzyk et al. 2000; Hwang 
et al. 2003; Wood et al. 2003). Subsequently, H2B K123 monoubiquitination is needed to control histone H3 K4 and $\mathrm{K} 79$ di- and trimethylation by a trans-histone pathway (Briggs et al. 2002; Dover et al. 2002; Ng et al. 2002; Sun and Allis 2002). It has been shown that the Rpt4 and Rpt6/Cim3 ATPase subunits of the 26S proteasome are recruited to chromatin by $\mathrm{H} 2 \mathrm{~B}$ ubiquitination and that mutations in Rpt4 and Rpt6/Cim3 that prevent ATPase function block H3 K4 and K79 methylation (Ezhkova and Tansey 2004). These data suggest that Rpt4 and Rpt6 might functionally link $\mathrm{H} 2 \mathrm{~B}$ ubiquitination to histone $\mathrm{H} 3$ K4 and K79 methylation (Ezhkova and Tansey 2004). A mutant of the Rpt1/Cim5 ATPase subunits of the 26S proteasome also shows defects in $\mathrm{H} 3 \mathrm{~K} 4$ trimethylation (Laribee et al. 2007). However, the precise mechanism by which the proteasome controls histone methylation needs further investigation.

Not4 is another E3 ubiquitin ligase that has been shown to be important for wild-type levels of H3 K4 trimethylation (Laribee et al. 2007; Mulder et al. 2007a). Not4 is one of the subunits of the CCR4/NOT complex, a multifunctional complex that has been implicated in transcriptional regulation and RNA deadenylation (Collart and Struhl 1994; Denis et al. 2001; Collart 2003; Collart and Timmers 2004; Mulder et al. 2007b). Although the precise role played by Not4 in the CCR4/ NOT complex remains to be determined, Not4 has been shown to have both positive and negative roles in transcriptional regulation and a positive role in transcriptional elongation (Collart 2003). In addition, the RING finger domain of yeast Not4 has been shown to be important for the induction of the ribonucleotide reductase $(R N R)$ gene family in response to DNA damage via $M M S$ and hydroxyurea and for E3 ubiquitin ligase activity (Mulder et al. 2007b). Until now, the mechanism by which Not4's RING finger domain acts to modulate histone H3 K4 methylation has been unclear and contradictory (Laribee et al. 2007; Mulder et al. 2007a).

These studies, as well as others, suggest that a proper balance of chromatin-modifying enzymes is needed for appropriate gene expression (Shi and Whetstine 2007; Lan et al. 2008). If this balance is disrupted, aberrant gene expression may lead to cell growth defects resulting in abnormal development and cancer (Shi and Whetstine 2007; Wang et al. 2007). Therefore, understanding the mechanism that regulates the balance between histone methyltransferases and demethylases is important and needs to be further explored.

In this study, we demonstrate that the protein level of the histone H3 K4 demethylase Jhd2 and its associated demethylase activity are controlled by Not4 through a polyubiquitin-mediated degradation process. We show that Jhd2 is polyubiquitinated in vitro and in vivo by Not 4 and degraded by the proteasome. In addition, in the absence of Not 4 or in the presence of a Not 4 mutant that lacks the RING finger domain, Jhd2 protein levels increase while histone $\mathrm{H} 3 \mathrm{~K} 4$ trimethylation levels decrease. Importantly, we determine that gene expression is modulated by the amount of Jhd 2 present and by $\mathrm{H} 3 \mathrm{~K} 4$ trimethylation levels. We also show that JARID1C, the human homolog of Jhd2, is ubiquitinated in vitro by human Not4. Our study suggests that polyubiquitination by E3 ubiquitin ligases and subsequent proteasomemediated degradation of histone demethylases is an important control mechanism in eukaryotes used to maintain a proper balance between histone methyltransferase and demethylase activities.

\section{Results}

High levels of Thd2 protein expression are required to detect in vivo H3 K4 demethylation

In $S$. cerevisiae, subtle but detectable changes in histone H3 K4 methylation have been observed when Jhd2 is overexpressed or deleted while in the presence of the $\mathrm{H} 3$ K4 methyltransferase Set1 (Liang et al. 2007). However, significant changes in H3 K4 methylation are observed only when Set1 is depleted in the presence of Jhd2 but not in the absence of Jhd2 (Seward et al. 2007). To explore the possibility that a proper balance of histone methyltransferases and demethylases is needed to detect significant changes in histone methylation, we wanted to determine if the amount of Jhd2 protein expressed affected in vivo H3 K4 methylation (Fig. 1B; Liang et al. 2007). Therefore, Flag epitope-tagged Jhd2 was expressed from CEN-based plasmids under the control of its own promoter (JHD2p) or overexpressed under the control of two constitutive yeast promoters, $A D H 1(A D H 1 p)$ and PYK1 (PYK1p), in a yeast strain containing wild-type $J H D 2$ and an endogenously MYC-tagged Set1. Jhd2 expression under the control of its own promoter is not detectable in wholecell lysates when immunoblotted with $\alpha$-Flag antibodies, while low levels of Jhd2 are detectable when it is expressed under the control of the $A D H 1$ promoter (Fig. 1B, cf. lanes 3 and 4). Interestingly, Jhd2 expressed from its own promoter or an $A D H 1$ promoter did not lead to a detectable decrease in histone $\mathrm{H} 3 \mathrm{~K} 4$ trimethylation levels (Fig. 1B). Expression of Jhd2 from the PYK1 promoter shows significantly increased amounts of protein expression relative to $\mathrm{Jh} d 2$ expressed from its endogenous promoter or an $A D H 1$ promoter (Fig. 1B, cf. lanes 3-5). In addition, the increased amount of Jhd2 expressed from the PYK1 promoter was sufficient to result in a substantial decrease in $\mathrm{H} 3 \mathrm{~K} 4$ trimethylation and subtle decreases in H3 K4 dimethylation (Fig. 1B [lane 5], D [lane 3]). This decrease in $\mathrm{H} 3 \mathrm{~K} 4$ trimethylation was not due to differences in histone $\mathrm{H} 3$ levels since immunoblots show equivalent levels of histone H3 (Fig. 1B). Furthermore, the amount of Jhd2 protein in a cell did not appear to alter the protein level of MYC-tagged Set1, suggesting that the observed decrease in H3 K4 trimethylation was a result of Jhd2's demethylase activity (Fig. 1B).

Because Jhd 2 expressed from its own promoter was not detectable in whole-cell lysates, immunoprecipitation assays were performed. Whole-cell lysates from strains expressing Flag-tagged Jhd2 from the $J H D 2, A D H 1$, or PYK1 promoters were normalized for protein levels, immunoprecipitated with $\alpha$-Flag resin, and immunoblotted 
A

Jhd2 \begin{tabular}{|l|l|l|l|l|l|l|}
\hline & Jmji & & PHD & & JmjC & \\
\hline 13 & 46 & 237 & 283 & 381 & 549 & 728
\end{tabular}

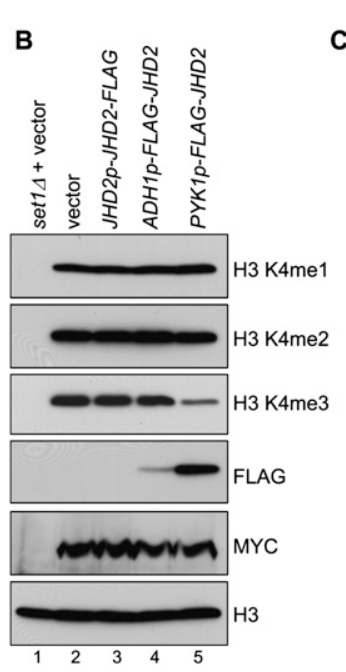

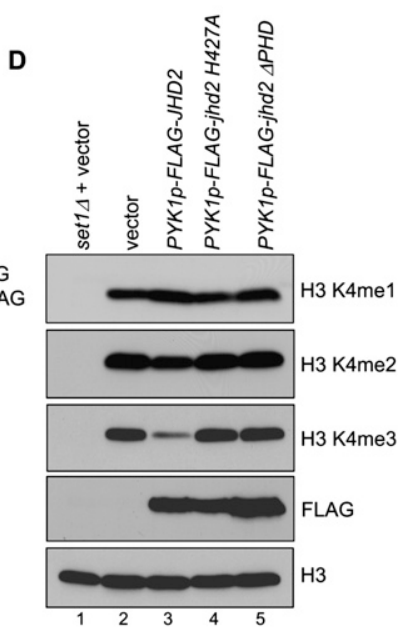

Figure 1. Histone $\mathrm{H} 3 \mathrm{~K} 4$ trimethylation is modulated by Jhd2 protein levels. (A) Schematic of Jhd2 illustrating positions of the JmjN, PHD, and JmjC domains. Numbers indicate amino acid positions. (B) Immunoblots of whole-cell lysates using H3 K4 mono-, di-, and trimethyl antibodies show the $\mathrm{H} 3$ K4 methylation status from yeast strains expressing the indicated constructs. An immunoblot probed with $\alpha-\mathrm{H} 3$ antibodies serves as a loading control. Flag-tagged Jhd2 and 3XMYC-Set1 proteins levels are assessed by immunoblots using $\alpha$ Flag or $\alpha$-MYC (9E10) antibodies. (C) Flag-tagged $J H D 2$ driven by its own promoter $(J H D 2 p)$, the $A D H 1$ promoter $(A D H 1 p)$, or the $P Y K 1$ promoter $(P Y K 1 p)$ was immunoprecipitated from whole-cell lysates. Detection of Flag-tagged Jhd2 is determined by immunoblotting with an $\alpha$-Flag antibody. Histone $\mathrm{H} 3$ is used as a loading control. $(D)$ Wholecell lysates generated from yeast strains expressing the indicated constructs are immunoblotted with H3 K4 mono-, di-, and trimethyl antibodies. As controls, $\alpha$-Flag and $\alpha-\mathrm{H} 3$ antibodies are used to determine Jhd2 and histone $\mathrm{H} 3$ proteins levels.

with $\alpha$-Flag antibodies (Fig. 1C). After immunoprecipitation, Jhd2 expressed from its own promoter was detectable (Fig. 1C, lane 3). Immunoprecipitation of Jhd2 also confirmed that Jhd 2 expression is substantially higher from a PYK1 promoter than from either its own promoter or when driven by the $A D H 1$ promoter (Fig. 1C). A histone $\mathrm{H} 3$ immunoblot confirmed that the protein lysates were normalized before immunoprecipitation (Fig. 1C). Although it was previously shown that overexpression of Jhd2 results in decreases in H3 K4 trimethylation, our results suggest that there is a critical amount of Jhd2 needed to offset the balance of histone methylation in favor of demethylase activity.

To determine if the decrease in $\mathrm{H} 3 \mathrm{~K} 4$ trimethylation was a consequence of Jhd2 demethylase activity, we generated a catalytically inactive mutant of Jhd2 (Jhd2 H427A) under the control of the PYK1 promoter (Liang et al. 2007). While overexpression of wild-type Jhd2 resulted in decreased $\mathrm{H} 3 \mathrm{~K} 4$ trimethylation, overexpression of Jhd2 H427A did not have a detectable effect on H3 K4 trimethylation (Fig. 1D, cf. lanes 3 and 4). These results suggest that the loss of $\mathrm{H} 3 \mathrm{~K} 4$ trimethylation observed when Jhd2 is overexpressed is due to its demethylase activity. It has been reported that the PHD domain of Jhd2 is not required for in vitro demethylase activity (Ingvarsdottir et al. 2007). We find that overexpressing Jhd2 lacking its PHD domain (Jhd2 $\triangle \mathrm{PHD}$ ) from a PYK1 promoter did not affect $\mathrm{H} 3 \mathrm{~K} 4$ trimethylation (Fig. 1D, lane 5). This is similar to what is observed when the catalytically inactive mutant Jhd2 H427A is overexpressed (Fig. 1D, cf. lanes 4 and 5). This is not due to differential expression of Jhd2, as near equal levels of Jhd2, Jhd2 H427A, and Jhd2 $\triangle$ PHD were detected when expressed in the $j h d 2 \Delta$ strain (Fig. 1D). Together, our in vivo results show that the PHD domain of Jhd2, and a particular amount of active Jhd2 protein, are needed to overcome Set1-mediated methylation before an observ- able loss of histone $\mathrm{H} 3 \mathrm{~K} 4$ trimethylation is globally detected. Our results suggest that a cell must monitor and control a critical balance between Jhd2 and Set1 for proper histone methylation. Therefore, Jhd2 is either expressed at very low levels from its own promoter and/ or Jhd2 protein levels are post-transcriptionally controlled by a protein degradation mechanism.

\section{The protein level of Jhd2 is controlled by the proteasome}

Since a particular amount of Jhd2 protein is needed to detect loss of H3 K4 trimethylation, and Jhd2 is expressed at very low levels, we wanted to determine if Jhd2 protein levels were post-translationally regulated by a proteasomemediated degradation pathway. Since Jhd2 is not detectable from whole-cell lysates when expressed from its own promoter, we wanted to determine if inhibiting the proteasome with MG132 would increase the amount of detectable Jhd 2 protein when expressed from its own promoter $(J H D 2 p)$. To determine this, Flag-tagged Jhd2 was expressed from its own promoter in a strain lacking both $J H D 2$ and PDR5 (jhd2Apdr5s). Yeast cells lacking the multidrug transporter Pdr5 are more sensitive to MG132 and are commonly used in combination with proteasome inhibitors to assess protein turnover/degradation (Ravid and Hochstrasser 2007).

After $30 \mathrm{~min}$ of treatment with either DMSO or MG132, whole-cell lysates were immunoblotted with $\alpha$-Flag antibodies to determine the amount of Jhd2 expressed. Similar to what was observed in Figure 1B, Jhd2 was not detectable in cells expressing Jhd2 from its own promoter when treated with DMSO (Fig. 2A, lanes 2,3). In contrast, when cells expressing Jhd 2 were treated with MG132, a dramatic increase in Jhd2 protein levels is observed (Fig. 2A, lanes 5,6). Histone H3 levels were monitored and used as a loading control. To confirm that 
A

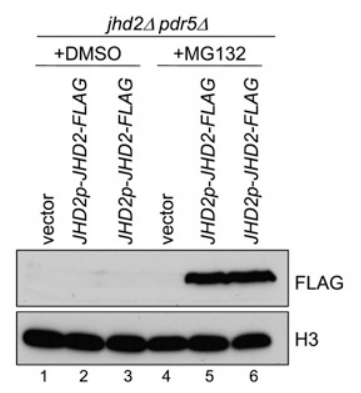

B

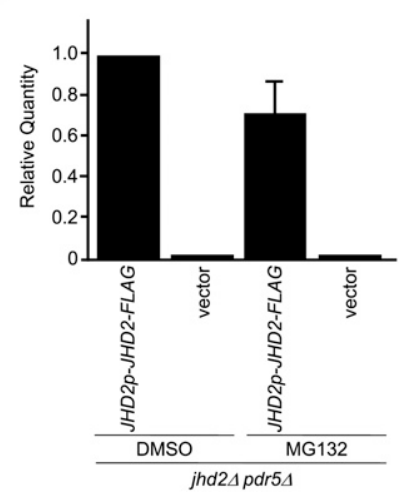

Figure 2. The proteasome mediates degradation of Jhd2. (A) A $j h d 2 \Delta p d r 5 \Delta$ yeast strain expressing JHD2-Flag driven by the $J H D 2$ promoter $(J H D 2 p)$ was treated with either DMSO or MG132. Immunoblots of whole-cell lysates are probed using an $\alpha$-Flag antibody to detect the amount of Jhd2 protein expressed. Histone $\mathrm{H} 3$ is used as a loading control. $(B)$ Transcript levels of JHD2 under the control of its endogenous promoter were examined after DMSO or MG132 treatment by quantitative real-time PCR.

the elevated levels of Jhd2 observed in Figure 2A are not due to increased transcription of $J H D 2$, we analyzed the levels of Jhd2 transcript (mRNA) in cells treated with either DMSO or MG132 by quantitative real-time PCR. Our results indicate that Jhd2 transcript levels did not increase in cells treated with MG132 relative to untreated cells, indicating that the observed increase in Jhd2 protein levels is due to inhibition of the proteasome and not increased transcription (Fig. 2B). Our results strongly indicate that Jhd2 protein levels are tightly controlled by the proteasome and are kept at a low level so that proper $\mathrm{H} 3 \mathrm{~K} 4$ trimethylation can take place.

\section{Thd2 is polyubiquitinated in vivo, and inhibition of Thd2 protein degradation decreases $\mathrm{H} 3 \mathrm{~K} 4$ trimethylation levels}

Post-translational modifications on histone demethylases have been suggested to modulate demethylase activity (Lan et al. 2008). However, post-translational modifications on yeast or mammalian histone demethylases that alter histone methylation levels have not been identified. Based on our results showing that the proteasome can control Jhd2 protein levels, we predicted that Jhd2 is post-translationally modified by ubiquitin in vivo and is thus targeted for degradation. Therefore, ubiquitinmediated degradation of Jhd2 would be an ideal mechanism to tightly control the balance between histone demethylase and histone methyltransferase activities in the cell. To test this idea and to identify a potential ubiquitination site on Jhd2, wild-type Flag-Jhd2, Flag-Jhd2 $\triangle \mathrm{PHD}$, and Flag-Jhd2 $\mathrm{N}$ - and C-terminal truncation mutants were expressed from an $A D H 1$ promoter in a $j h d 2 \Delta$ strain (see Fig. 1A for positions of Jhd2 mutations represented in Fig 3A). Whole-cell lysates derived from the indicated strains were immunoprecipitated with

$\alpha$-Flag resin and immunoblotted for Flag-Jhd2 and ubiquitin using $\alpha$-Flag and $\alpha$-Ubiquitin (Ub) antibodies. We clearly observe that Jhd2 is polyubiquitinated when isolated from cells (Fig. 3A). Importantly, polyubiquitination was specific to Jhd2 since no polyubiquitination was detected in a $j h d 2 \Delta$ strain expressing empty vector (Fig. $3 \mathrm{~A}$, lane 1). Interestingly, all of the Jhd 2 mutants analyzed also appear to be polyubiquitinated (Fig. 3A, lanes 3-8). Further work will be needed to determine if one or more ubiquitination sites are involved in degrading Jhd2. This would not be unexpected, as it is known that E3 ubiquitin ligases can ubiquitinate more than one lysine residue on a substrate (Pickart 2001; Peng et al. 2003).

To confirm that polyubiquitination of Jhd2 was not artificially caused by overexpression of Jhd2, Jhd2 under the control of its own promoter was expressed in a $j h d 2 \Delta p d r 5 \Delta$ strain. In order to capture polyubiquitinated Jhd2 that is under the control of its own promoter, cells expressing Jhd2-Flag were treated with or without MG132 for $30 \mathrm{~min}$. Whole-cell lysates were prepared in the presence of the thiol-alkylating agent $\mathrm{N}$ ethylmaleimide (NEM) in order to inhibit deubiquitination of Jhd2 upon cell lysis. Strikingly, robust levels of polyubiquitinated Jhd 2 are detected from cells expressing Jhd2 from its own promoter when treated with both MG132 and NEM (Fig. 3B). Since Jhd2 is highly polyubiquitinated after MG132 and NEM treatment, less unmodified Jhd2 was able to be immunoprecipitated from MG132- and NEM-treated cells than from cells treated with only NEM (Fig. 3B, cf. lanes 3 and 4). In addition, polyubiquitination of Jhd2 was specific since it was not detected in wild-type or $j h d 2 \Delta p d r 5 \Delta$ cells expressing empty vector. Furthermore, polyubiquitination of Jhd2 was not due to the Flag tag since Jhd2 tagged with HA was also observed to be polyubiquitinated in a similarly robust manner (data not shown).

To address the possibility that episomal expression of Jhd2 expressed from its own promoter resulted in artificial polyubiquitination of Jhd2, a 3XFlag tag was engineered at the $\mathrm{C}$ terminus of Jhd 2 at its endogenous locus. To sensitize Pdr5-positive cells for better uptake of MG132, wild-type and the integrated Jhd2-3XFlag strains were treated as described previously (Liu et al. 2007). Whole-cell lysates were prepared in the presence of NEM to prevent deubiquitination of Jhd2. Robust levels of polyubiquitinated Jhd 2 were detected in cells expressing Jhd2-3XFlag at its endogenous locus that were treated with NEM and MG132 (Fig. 3C, lane 3). MG132 treatment was needed to detect polyubiquitinated Jhd2 (Fig. 3C, cf. lanes 2,3). Together, our results unequivocally indicate that Jhd2 is polyubiquitinated under physiologically expressed conditions and when it is overexpressed.

Since inhibition of the proteasome by MG132 results in higher levels of Jhd2 protein, we wanted to determine if proteasome inhibition would affect global H3 K4 trimethylation levels in a Jhd2-dependent manner. To test this, Jhd2 was deleted in a strain expressing MYC-tagged Set1 at its endogenous locus. To sensitize cells to MG132 treatment, wild-type and $j h d 2 \Delta$ strains were treated as described previously (Liu et al. 2007). MG132 treatment of 


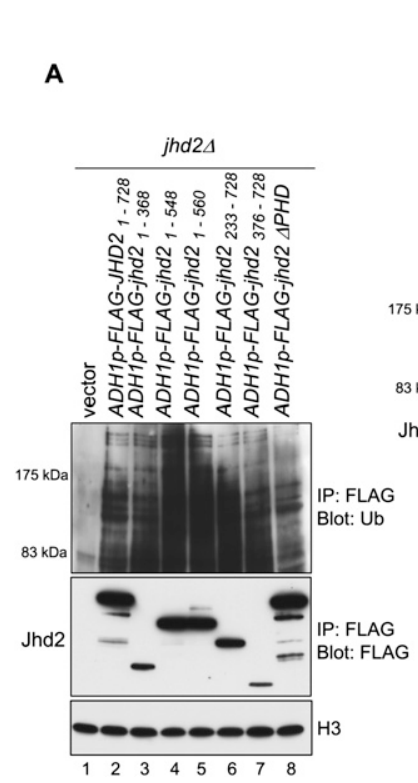

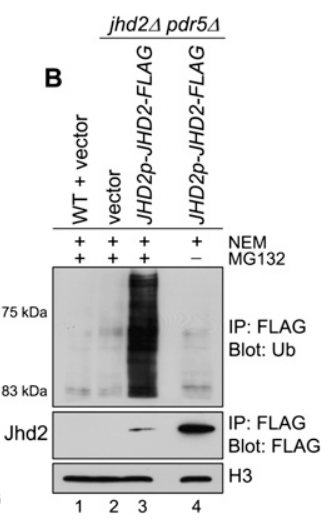
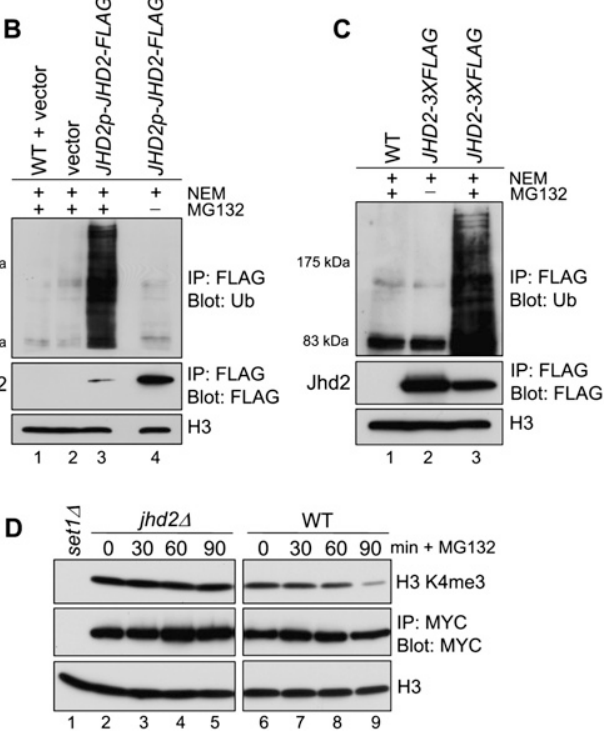

Figure 3. Jhd 2 is polyubiquitinated in vivo. $(A)$ $J H D 2$ and $j h d 2$ mutant constructs were expressed from an $A D H 1$ promoter (ADH1p). Subscripted numbers represent amino acid positions and protein size of Jhd2 mutants. Whole-cell extracts expressing the indicated Jhd2 constructs were immunoprecipitated with $\alpha$-Flag resin. Detection of Flag-Jhd2 and Flag-Jhd2 mutants are determined by immunoblotting with an $\alpha$-Flag antibody. Polyubiquitination of FLAG-Jhd2 is detected with an $\alpha$ Ubiquitin antibody. Histone $\mathrm{H} 3$ immunoblots shows equivalent amounts of lysates were used for immunoprecipitation. (B) JHD2 driven by its own promoter $(J H D 2 p)$ was immunoprecipitated from whole-cell lysates of strains expressing the indicated constructs. Strains were treated with or without MG132 before cell lysis, and whole-cell lysates were prepared in an extraction buffer containing NEM before immunoprecipitation. Detection of Flag-Jhd2 is determined by immunoblotting with $\alpha$-Flag antibody. Polyubiquitinated Flag-Jhd2 is detected with $\alpha$-Ubiquitin antibody. A histone H3 immunoblot serves as a loading control. $(C)$ JHD2-3XFlag expressed from its endogenous locus was treated with or without MG132 before immunoprecipitation. Whole-cell lysates were prepared in the presence of NEM to inhibit deubiquitination. Detection of Jhd2-3XFlag is determined by immunoblotting with an $\alpha$-Flag antibody. Polyubiquitinated Jhd3-3XFlag is detected with an $\alpha$-Ubiquitin antibody. A histone $\mathrm{H} 3$ immunoblot serves as a loading control, and untagged JHD2 serves as a control for immunoprecipitation. $(D)$ Wild-type and $j h d 2 \Delta$ yeast strains in which 3XMYC-Set1 was tagged at its endogenous locus were treated with MG132 and samples were taken at the indicated time points. Immunoblots of whole-cell lysates are probed with $\alpha-\mathrm{H} 3 \mathrm{~K} 4$ trimethyl and $\alpha-\mathrm{H} 3$ antibodies. 3XMYC-Set1 was detected by immunoprecipitation followed by immunoblotting with an $\alpha$-MYC antibody.

the wild-type strain resulted in substantially decreased levels of H3 K4 trimethylation after 90 min (Fig. 3D, lane 9). In a different strain, a similar decrease in H3 K4 trimethylation was obtained in a $j h d 2 \Delta p d r 5 \Delta$ strain expressing Jhd2 (Supplemental Fig. S1). Importantly, the observed reduction of $\mathrm{H} 3 \mathrm{~K} 4$ trimethylation required the presence of Jhd2, since inhibition of the proteasome by MG132 in a $j$ hd2 $\Delta$ strain had no apparent effect on H3 K4 trimethylation (Fig. 3D, lanes 2-5). Changes in H3 K4 trimethylation were not due to decreased levels of Set1 expression or changes in histone $\mathrm{H} 3$ levels since immunoblots showed that MYC-tagged Set1 is similarly expressed in both strains and showed nearly equivalent loading (Fig. 3D). Together, our results show that Jhd2 expressed from its endogenous locus is controlled by being post-translationally modified by ubiquitin and degraded by the proteasome. Inhibition of Jhd2 degradation in turn leads to increased Jhd2 protein levels and decreased levels of $\mathrm{H} 3 \mathrm{~K} 4$ trimethylation.

\section{Thd2 and Not4 genetically interact to mediate proper levels of $\mathrm{H3} \mathrm{K} 4$ trimethylation}

Since Jhd2 is ubiquitinated in vivo and degraded by the proteasome, we wanted to identify the enzyme responsible for ubiquitinating Jhd2 using a genetic approach. We predicted that Jhd2 would not be ubiquitinated in a yeast strain that lacked this enzyme and that this strain would have decreased levels of trimethylated H3 K4 because of increased amounts of Jhd 2 protein. Yeast strains that are deleted for both JHD2 and the enzyme that ubiquitinates Jhd2 would be expected to restore H3 K4 trimethylation. Based on the above criteria, Rad6, an E2 ubiquitinconjugating enzyme that, when deleted, disrupts H3 K4 mono-, di-, and trimethylation, and Not4, an E3 RING finger domain ubiquitin ligase that when deleted decreases global H3 K4 trimethylation, were chosen as the most likely candidates to ubiquitinate Jhd2 (Sun and Allis 2002; Wood et al. 2003; Shahbazian et al. 2005; Laribee et al. 2007).

The H3 K4 mono-, di-, and trimethylation levels in

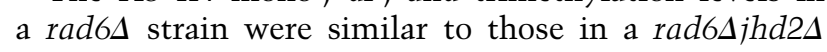
strain as indicated by immunoblotting with methylspecific H3 K4 antibodies (Fig. 4A, lanes 4,5). The levels of histone $\mathrm{H} 3$ and $\mathrm{H} 3 \mathrm{~K} 36$ trimethylation were used as controls (Fig. 4A). To show specificity of K4 and K36 methyl-specific antibodies, set $1 \Delta$ and set $2 \Delta$ strains were used (Fig. 4A). This suggests that Rad6 does not modulate Jhd2 protein levels by polyubiquitination. As previously published, a not $4 \Delta$ strain exhibited a decrease in H3 K4 trimethylation (Laribee et al. 2007; Mulder et al. 2007a). However, as predicted based on a genetic interaction, wild-type levels of $\mathrm{H} 3 \mathrm{~K} 4$ trimethylation were restored in a not $4 \Delta j h d 2 \Delta$ double deletion strain (Fig. 4B, lanes 4,5 ). No changes in global levels of H3 K4 mono- or dimethylation were detected in $j h d 2 \Delta$, not $4 \Delta$, or not $4 \Delta j h d 2 \Delta$ strains, and histone $\mathrm{H} 3$ immunoblots indicated equal loading (Fig. 4B). These results suggest that Not4 is the E3 ubiquitin ligase controlling Jhd2 protein levels. 
Figure 4. Not4 and Jhd2 genetically interact and modulate $\mathrm{H} 3 \mathrm{~K} 4$ trimethylation. (A) Histone H3 K4 and K36 methylation levels were analyzed from whole-cell lysates from the indicated strains by immunoblotting using H3 K4 mono-, di-, and trimethylation and $\mathrm{H} 3 \mathrm{~K} 36$ trimethylation antibodies. Histone $\mathrm{H} 3$ is used as loading control. $(B)$ Histone H3 K4 methylation status of the indicated strains was determined using the indicated H3 K4 methyl-specific and histone $\mathrm{H} 3$ antibodies. $(C)$ Whole-cell lysates from the indicated yeast deletion strains are analyzed for $\mathrm{H} 3 \mathrm{~K} 4$ methylation. Histone H3 levels and expression of Jhd2-Flag and Jhd2-Flag mutants are verified by $\alpha-\mathrm{H} 3$ and $\alpha$-Flag antibody immunoblots.
A

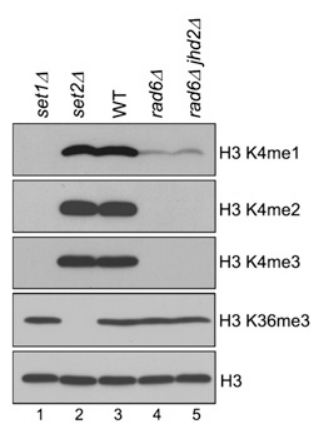

B

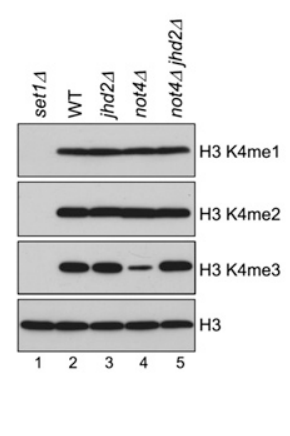

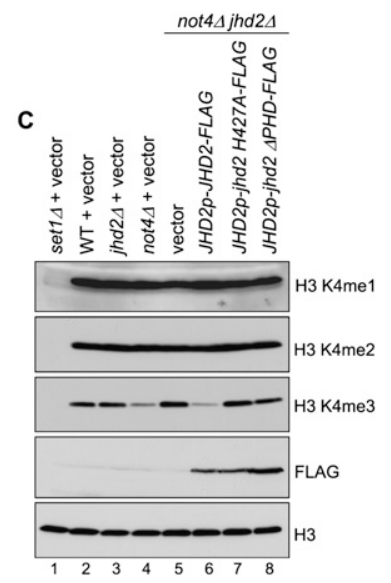

To show that the restoration of $\mathrm{H} 3 \mathrm{~K} 4$ trimethylation in the not $4 \Delta$ jhd2 $\Delta$ strain was the result of losing the demethylase activity of Jhd2 and to confirm the importance of the PHD domain for Jhd2 demethylase activity, wild-type Jhd2, Jhd2 H427A, and Jhd2 $\triangle$ PHD were expressed under the control of the JHD2 promoter $(J H D 2 p)$ in the not $4 \Delta j h d 2 \Delta$ strain. Expression of wildtype Jhd2 in the not $4 \Delta$ jhd $2 \Delta$ strain resulted in decreased levels of $\mathrm{H} 3 \mathrm{~K} 4$ trimethylation similar to what is observed in a not $4 \Delta$ strain (Fig. 4C, lanes 4,6). As expected, expression of either Jhd2 H427A or Jhd2 $\triangle$ PHD did not result in a detectable decrease $\mathrm{H} 3 \mathrm{~K} 4$ trimethylation (Fig. 4C, lanes 7,8). Changes in $\mathrm{H} 3 \mathrm{~K} 4$ trimethylation levels are specific to the protein level and demethylase activity of Jhd2 since equal loading of histone $\mathrm{H} 3$ is shown by $\mathrm{H} 3$ immunoblots. Unlike in cells expressing Not4, Jhd2 expressed from its own promoter is detected in wholecell lysates in the absence of Not4 (cf. Figs. 1B [lane 3] and 4C [lane 6]). Jhd2 H427A and Jhd2 $\triangle$ PHD are also detected in the absence of Not4 (Fig. 4C, lanes 7,8). Using these genetic approaches, we showed that Jhd 2 and Not4 are both necessary to mediate proper levels of H3 K4 trimethylation. More importantly, our data suggest that the histone demethylase Jhd2 is a potential in vivo substrate for Not4-mediated polyubiquitination.

\section{The RING finger domain of Not4 mediates in vivo $\mathrm{H3}$ K4 trimethylation and Thd2 protein levels}

It has recently been determined that Ubc4 is the E2 ubiquitin conjugating enzyme for Not4 and that the RING finger domain of Not4 is needed to mediate ubiquitination of itself and one identified substrate (Albert et al. 2002; Panasenko et al. 2006; Mulder et al. 2007a). In addition, an N-terminal truncation of Not4 that removes the RING finger domain cannot restore $\mathrm{H} 3$ K4 trimethylation in a not4s strain (Laribee et al. 2007). To determine if the RING finger domain of Not4 by itself is important for proper levels of H3 K4 trimethylation, we constructed Not4 deletion mutants lacking either the RING finger domain or the putative RNA recognition motif (RRM) (Fig. 5A). Wild-type Not4 and both Not4 deletion mutants were HA-tagged and expressed from
Not4's own promoter (NOT4p) in a not4d strain. While expression of Not 4 and Not $4 \Delta R R M$ in a not $4 \Delta$ strain resulted in restoration of wild-type levels of $\mathrm{H} 3 \mathrm{~K} 4$ trimethylation, expression of either empty vector or Not4 $\triangle$ RING did not restore H3 K4 trimethylation (Fig. $5 \mathrm{~B}$, cf. lanes 3,5 and 4,6). Immunoblots using $\alpha$-HA and $\alpha-\mathrm{H} 3$ antibodies indicated that all Not 4 constructs were equivalently expressed and equally loaded (Fig. 5B). To determine if the RING finger domain of Not4 was

A
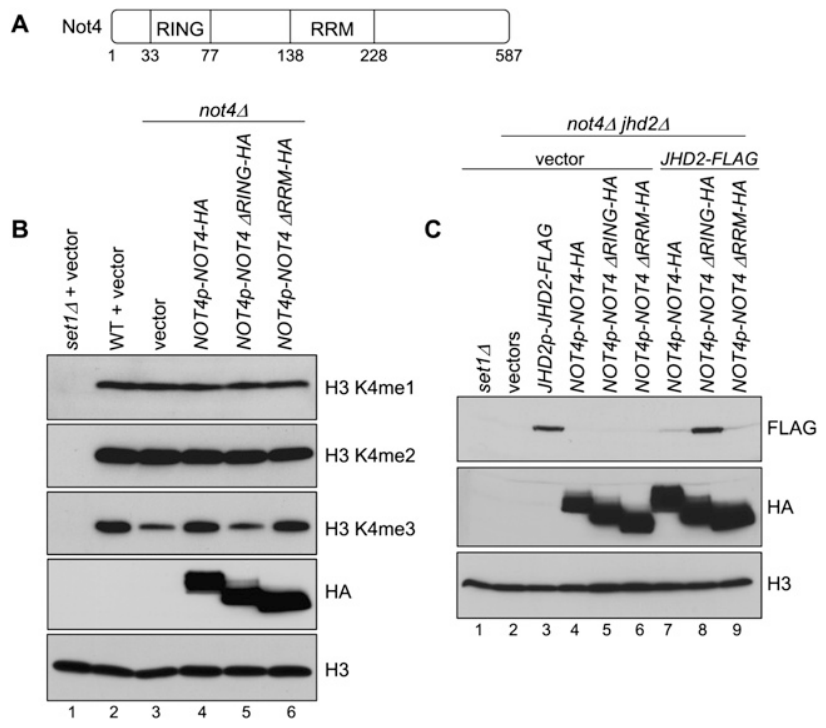

Figure 5. The RING finger domain of Not4 is required for $\mathrm{H3}$ K4 trimethylation and Jhd2 protein stability. (A) Schematic of Not4 illustrating positions of RING and RRM domains. Numbers indicate amino acid positions. $(B)$ Whole-cell lysates from yeast strains expressing the indicated constructs were analyzed for histone $\mathrm{H} 3 \mathrm{~K} 4$ methylation by immunoblotting with $\mathrm{H} 3 \mathrm{~K} 4$ mono-, di-, or trimethyl-specific antibodies. Histone H3 levels and expression levels of Not4-HA and Not4-HA deletion mutants were verified using $\alpha-\mathrm{H} 3$ and $\alpha-\mathrm{HA}$ antibodies. $(C)$ Jhd2 expression was analyzed in a not $4 \Delta j h d 2 \Delta$ strain expressing the indicated Not 4 constructs with a $\alpha$-Flag antibody. HA-tagged Not4 and Not4 deletion mutants are detected with a $\alpha-\mathrm{HA}$ antibody. Histone $\mathrm{H} 3$ is used as a loading control. 
important for maintaining wild-type levels of Jhd2 protein, Jhd2 was coexpressed with wild-type Not4, Not4 $\Delta$ RING, or Not4 $\Delta$ RRM. All constructs were expressed from their own promoters in a not $4 \Delta$ jhd $2 \Delta$ strain. Coexpression of Jhd2 with Not4 $\Delta$ RING resulted in a significant increase of Jhd2 protein levels relative to when Jhd2 was expressed with wild-type Not4 or Not4 $\Delta$ RRM (Fig. 5C, cf. lanes 7-9). Wild-type Jhd2 and the indicated Not 4 constructs were expressed individually as controls (Fig. 5C, lanes 3-6). Together, our results identify Jhd 2 as a potential substrate for Not 4 and indicate that the RING finger domain of Not4 mediates both H3 $\mathrm{K} 4$ trimethylation and Jhd2 protein levels. This suggests that Jhd 2 may be a novel substrate for Not4-mediated polyubiquitination.

Yeast and human Not4 ubiquitinate yeast and human histone demethylases, Jhd2 and JARID1C

Since we determined that Jhd2 can be ubiquitinated in vivo and that the RING domain of Not4 is required to control Jhd2 protein and H3 K4 trimethylation levels, we predicted that Not4 would polyubiquitinate Jhd2 in vivo and in vitro. To test this, yeast Not4 and Jhd2 were expressed in Escherichia coli and purified for use in an in vitro ubiquitination assay. In the presence of purified ubiquitin-activating enzyme Ube1 (E1) and ubiquitinconjugating enzyme Ubc4 (E2), Not4 (E3) is able to mediate polyubiquitination of CBP-tagged Jhd2 in 30 min as indicated by the accumulation of high molecular weight species detected in $\alpha$-CBP immunoblots (Fig. 6A). Polyubiquitination of Jhd2 did not occur when Ube1, Ubc4, or Not4 were excluded from the ubiquitination reaction. As an additional control, inclusion of the alkylating agent NEM, which inhibits the activity of E2 ubiquitin-conjugating enzymes, in the reaction also prevented polyubiquitination of Jhd2 (Fig. 6A). As a control for nonspecific ubiquitination by Not4, CBP-tagged Bre2, a component of the yeast Set 1 complex, was included in a similar in vitro ubiquitination assay (Supplemental Fig. S2A,B). Not4 does not polyubiquitinate Bre2 under similar conditions used for detecting polyubiquitinated Jhd2, suggesting that Jhd2 is a bona fide substrate of Not4 (Supplemental Fig. S2A,B).

To test if Jhd2 is a relevant substrate for Not4-mediated ubiquitination in vivo, wild-type and not $4 \Delta$ strains expressing Jhd2-3XFlag from its endogenous locus were sensitized to MG132 treatment as described (Liu et al. 2007). After $30 \mathrm{~min}$ incubation with or without MG132, whole-cell lysates were prepared in the presence of NEM to prevent artificial deubiquitination of Jhd2. Immunoprecipitation of whole lysates with $\alpha$-Flag resin and immunoblotting with $\alpha$-Flag and $\alpha$-Ubiquitin antibodies were used to detect unmodified Jhd2 and polyubiquitinated Jhd2. Polyubiquitination of Jhd2 was detected in the presence of Not4; however, in the absence of Not4, polyubiquitination of Jhd2 was eliminated (Fig. 6B, cf. lanes 3 and 4). In addition, Flag-tagged Jhd2 was polyubiquitinated when expressed from the $A D H 1$ promoter in jhd2s, but polyubiquitination of Jhd2 was greatly decreased when expressed in a not $4 \Delta$ jhd2 $\Delta$ strain (Supplemental Fig. S3, cf. lanes 4,5). Our in vitro and in vivo biochemical studies show that Jhd2 is a novel substrate for the E3 ligase activity of Not4 and is the second identified substrate for Not4. Furthermore, Not4 is needed to control the levels of Jhd2 to maintain appropriate levels of $\mathrm{H} 3 \mathrm{~K} 4$ trimethylation in vivo.

Because Jhd2 and Not4 are conserved in humans, we wanted to determine if JARID1C, the human homolog of yeast Jhd2, would be subject to ubiquitination by human Not4 (Hanzawa et al. 2001). To test this, bacterial constructs that express the first 700 amino acids (1-700 amino acids) of JARID1C or human Not4 were generated and expressed in E. coli and purified for use in an in vitro
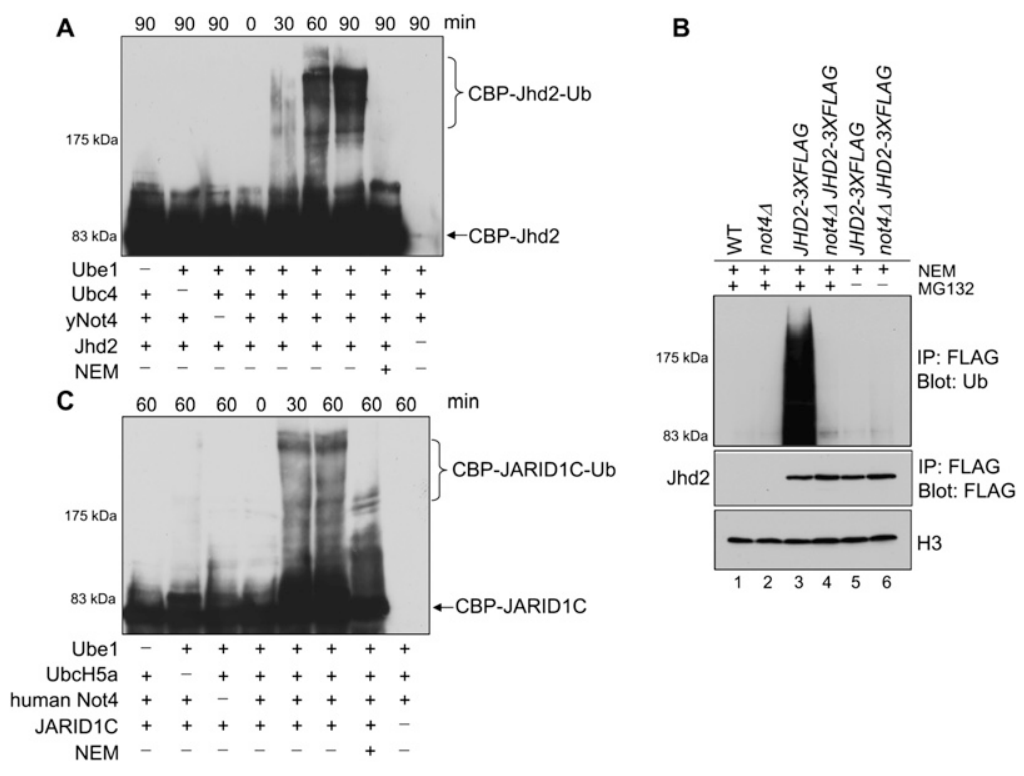

Figure 6. Thd2 is polyubiquitinated by the E3 ubiquitin ligase Not4 in vitro and in vivo. $(A)$ In vitro ubiquitination assays were performed by incubating Jhd2 and Not4 with Ube1, Ubc4, ATP, and free ubiquitin for 0, 30, 60, and $90 \mathrm{~min}$. Polyubiquitinated and unmodified CBP-tagged Jhd2 were detected with a $\alpha$ CBP antibody. NEM is used as a control to show specificity by inhibiting the ubiquitination reaction. (B) JHD2-3XFlag expressed from its endogenous locus was treated with or without MG132 and immunoprecipitated from the indicated strains. Polyubiquitination and expression of Jhd2 were confirmed with $\alpha$ Ubiquitin and $\alpha$-Flag antibodies. Immunoblots of histone $\mathrm{H} 3$ from whole-cell lysates indicated equivalent amounts of lysates used for immunoprecipitation assays. NEM was added to the lysis buffer to inhibit deubiquitination. $(C)$ In vitro ubiquitination assays were performed by incubating JARID1C $\mathrm{C}_{1-700}$ and hNot4 (human E3) with Ubeland UbcH5a in the presence of ATP and ubiquitin. Polyubiquitinated and unmodified JARID1C $_{1-700}$ were detected by immunoblotting with an $\alpha$-CBP antibody. NEM was used as a control to show specificity and inhibit the ubiquitination reaction. 
ubiquitination assay. In the presence of Ube1 (E1) and UbcH5a (E2 and the human homolog of Ubc4), human Not4 was able to polyubiquitinate JARID1C (Fig. 6C). Polyubiquitinated JARID1C was not detected when the E2 conjugating enzyme was inhibited by NEM or when Ube1, UbcH5a, or hNot4 were missing from the ubiquitination reaction (Fig. 6C). These in vitro results demonstrate that human demethylases are capable of being ubiquitinated and suggest that human Not4 or other E3 ubiquitin ligases may be important in regulating human histone demethylases and histone methylation levels in a manner similar to what we showed in yeast.

\section{Not4 and Thd2 modulate GUA1 expression and gene-specific H3 K4 trimethylation}

Since we established that polyubiquitin-mediated degradation of Jhd 2 by Not 4 modulates in vivo H3 K4 trimethylation, we wanted to determine if gene expression levels would also be modulated by Jhd2 and H3 K4 trimethylation levels. To test this idea, we examined the gene expression of GUA1, a GMP synthetase, which was recently determined by microarray analysis to be a gene putatively regulated by Not4 (Cui et al. 2008). The level of GUA1 expression was first analyzed in wild-type, $j h d 2 \Delta$, not $4 \Delta$, and not $4 \Delta j h d 2 \Delta$ strains expressing either empty vector, Not4-HA, Jhd2-Flag, or jhd2 H427A-Flag expressed from their own promoters by quantitative realtime PCR analysis (Fig. 7A; see Supplemental Table S3 for quantitative values; see Supplemental Fig. S4A for primer positions). GUA1 expression was decreased 2.5-fold in a not $4 \Delta$ strain compared with wild-type, but was restored to wild-type levels in a not $4 \Delta$ jhd2 2 strain (Fig. 7A; Supplemental Table S3). No significant change in GUA1 expression was observed between wild-type and $j h d 2 \Delta$ strains (Fig. 7A; Supplemental Table S3). When Jhd2-Flag was expressed in the not $4 \Delta j h d 2 \Delta$ strain, GUA1 expression was decreased 2.13-fold, similar to what is observed in not44 (Fig. 7A; Supplemental Table S3). No significant change in GUA1 expression was observed between a wild-type strain, $j h d 2 \Delta$, and a not $4 \Delta j h d 2 \Delta$ strain expressing either Not4-HA or the catalytically inactive $j h d 2$ H427A-Flag (Fig. 7A; Supplemental Table S3). Similar gene expression results were obtained from strains grown in rich media (Supplemental Fig. S4B). In addition, GUA1 expression was analyzed by quantitative real-time PCR in wild-type and set1A strains (Fig. 7B). GUA1 expression was decreased 2.04-fold in the set $1 \Delta$ strain relative to the wild-type strain (Fig. 7B; Supplemental Table S4). For all gene expression analysis, ACT1 (actin) mRNA was used as an internal control. Taken together, these results suggest that Set1-mediated H3 K4 trimethylation is important for wild-type levels of GUA1 expression.

To determine if $\mathrm{H} 3 \mathrm{~K} 4$ trimethylation at the GUA1 locus is affected in each of these strains, chromatin immunoprecipitations (ChIPs) were performed using H3 K4 di- and trimethyl antibodies. ChIP eluates were analyzed by quantitative real-time PCR using TaqMan primer and probe pairs designed to analyze the $5^{\prime}$ and $3^{\prime}$ ends of GUA1 and normalized to histone H3 levels (see
Figure 7. H3 K4 trimethylation is important for wild-type expression of GUA1. $(A, B)$ Expression of GUA1 was determined in the indicated strains by quantitative real-time PCR analysis. ACT1 (Actin) expression was used as an internal control to normalize expression levels. Data in $A$ were analyzed from six biological repeats with four technical repeats each. Data in $B$ were analyzed from three biological repeats with three technical repeats each. $(C, D)$ ChIP from the indicated strains were performed using antibodies specific to H3 K4 dimethylation, trimethylation, and histone H3. ChIP eluates were analyzed by quantitative real-time PCR using $5^{\prime}$ and $3^{\prime}$ GUA1 gene-specific TaqMan primer sets and normalized to histone H3 levels. Data in $C$ and $D$ were analyzed from three biological repeats with three technical repeats each. See Supplemental Tables S3-S6 for quantitative values of gene expression and ChIP analysis. $(E)$ In the depicted model, the histone demethylase Jhd 2 is polyubiquitinated by Not 4 and Ubc4. Upon polyubiquitination, Jhd2 is targeted for proteasome-mediated degradation that permits the Set1 H3 K4 methyltransferase complex (Set1C) to maintain wild-type levels of histone methylation resulting in normal levels of gene transcription. In the absence of Not4, Jhd2 ubiquitination and subsequent degradation by the proteasome is greatly decreased. This leads to an increase in Jhd2 protein levels and its associated demethylase activity, which offsets the methyltransferase activity mediated by Set1C resulting in decreased H3 K4 trimethylation and gene expression.
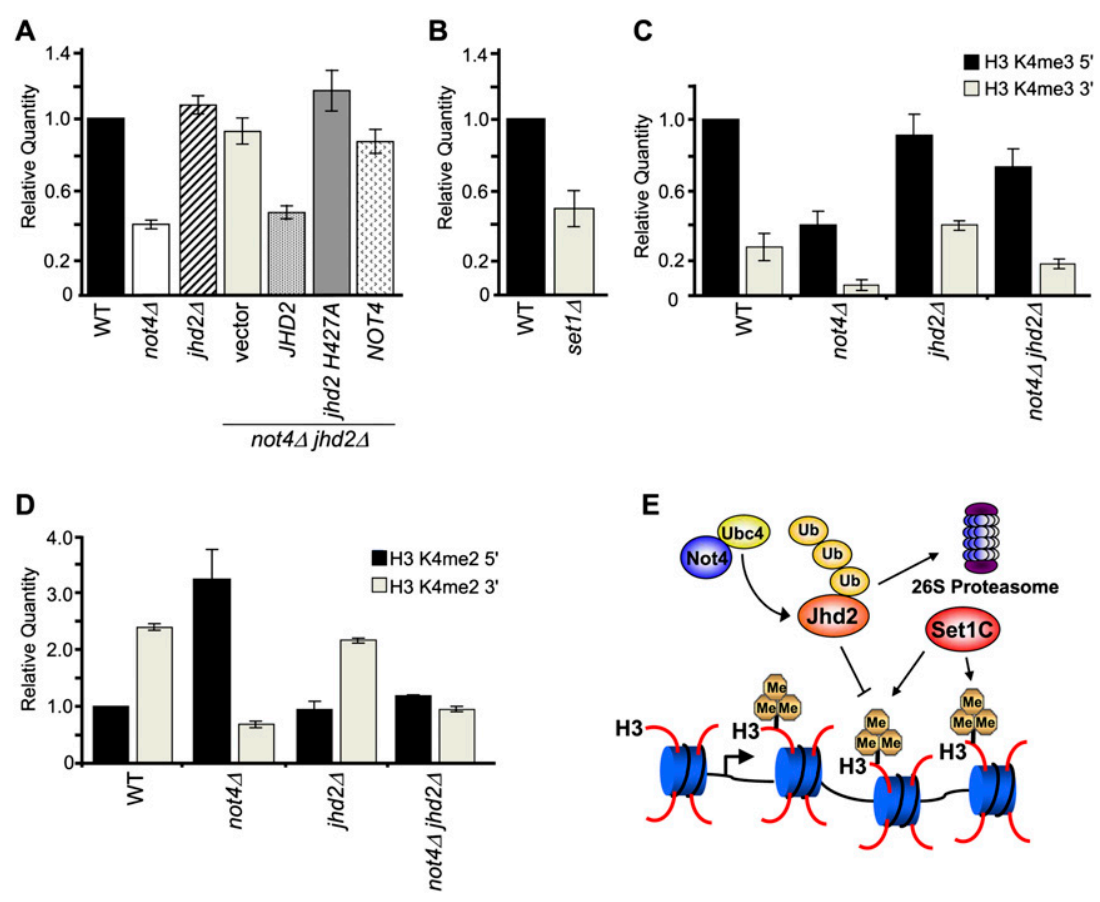
Supplemental Tables S5, S6 for quantitative values; see Supplemental Fig S4A for primer positions). As expected, GUA1 exhibits high levels of $\mathrm{H} 3 \mathrm{~K} 4$ trimethylation at the $5^{\prime}$ end and decreased levels of H3 K4 trimethylation at the $3^{\prime}$ end in all strains (Fig. 7C; Supplemental Table S5; Supplemental Fig. S5A,B). However, H3 K4 trimethylation is decreased 2.5 -fold in the not $4 \Delta$ strain at the $5^{\prime}$ end of GUA1 and is almost restored in the not $4 \Delta j h d 2 \Delta$ strain when compared with wild-type cells (Fig. 7C; Supplemental Table S5; Supplemental Fig. S5A). In a similar manner, $\mathrm{H} 3 \mathrm{~K} 4$ trimethylation is decreased 4.5 -fold in the not $4 \Delta$ strain at the $3^{\prime}$ end of GUA1 and is partially restored in the not $4 \Delta$ jhd2 $\Delta$ strain (Fig. 7C; Supplemental Table S5; Supplemental Fig. S5B). Interestingly, we observe that H3 K4 dimethylation is increased 3.2-fold in the not $4 \Delta$ strain at the $5^{\prime}$ end of GUA1 and that dimethylation is restored to wild-type levels in the not $4 \Delta$ jhd2d strain (Fig. 7D; Supplemental Table S6; Supplemental Fig. S5C). The increase in 5' H3 K4 dimethylation in a not $4 \Delta$ strain is likely due to the consequence of decreased 5' H3 K4 trimethylation levels. In contrast, 5' H3 K4 dimethylation levels in a not $4 \Delta$ jhd2 2 strain does not change since 5' H3 K4 trimethylation levels are nearly restore wild-type levels. Intriguingly, H3 K4 dimethylation levels at the 3 ' end of GUA1 decrease in a not $4 \Delta$ strain but do not appear to be restored in a not $4 \Delta j h d 2 \Delta$ strain (Fig. 7D; Supplemental Table S6; Supplemental Fig. S5D). H3 K4 dimethylation in a $j h d 2 \Delta$ strain appears to have little, if any, effect on $\mathrm{H} 3 \mathrm{~K} 4$ dimethylation at the 5' or 3' ends of GUA1 (Fig. 7D; Supplemental Table S6; Supplemental Fig. S5C, D). Similar ChIP results were obtained by PCR and gel analysis (Supplemental Fig. S4C).

Together, these results show that GUA1 gene expression is modulated by the levels of $\mathrm{H} 3 \mathrm{~K} 4$ trimethylation mediated by Not 4 and Jhd2. Overall, our results indicate that polyubiquitination of Jhd2 by Not4 is a way to control Jhd2 protein levels and its associated demethylase activity so that appropriate levels of $\mathrm{H} 3 \mathrm{~K} 4$ methylation and gene expression occur in a cell (Fig. 7E).

\section{Discussion}

In this study, we determined for the first time that a histone demethylase is polyubiquitinated in vivo, which leads to degradation by the proteasome (Fig. 7E). More importantly, we show that polyubiquitin-mediated degradation of Jhd2 is necessary to keep the levels of Jhd2 protein expression low so that its demethylase activity does not disrupt global and gene-specific histone H3 K4 trimethylation and gene expression levels. We also identified Not4 as a RING finger domain E3 ubiquitin ligase that mediates Jhd2 polyubiquitination in vivo and in vitro. Furthermore, in the absence of Not4 or during inhibition of the proteasome, Jhd2 protein levels are significantly increased resulting in decreased H3 K4 trimethylation. Therefore, a critical role for Not4 and the proteasome is to constantly monitor and maintain low Jhd2 protein levels so that aberrant histone demethylation and gene expression do not take place. Finally, we show that human Not4 can polyubiquitinate JARID1C, a human homolog of Jhd2. Therefore, other yeast and human E3 ubiquitin ligases are likely playing key roles in maintaining the proper balance between demethylase and histone methyltransferase activities.

\section{Regulating and controlling histone demethylase activity}

Histone demethylases are expressed in a temporal and tissue-specific manner in multicellular organisms, and their expression patterns can be altered by various environmental cues (Lan et al. 2008). In addition, histone demethylase activity can be regulated by associated proteins (Shi et al. 2005). If histone demethylases are aberrantly expressed or regulated, this can result in devastating consequences such as human cancers or developmental defects leading to mental retardation (Shi and Whetstine 2007; Wang et al. 2007; Cloos et al. 2008). Therefore, how histone demethylases are regulated at the gene or protein level needs further investigation and is essential for understanding their biological function.

Interestingly, mammalian histone demethylases have been shown to be post-translationally modified by phosphorylation (Lan et al. 2008). However, no biological relevance has been attributed to a phosphorylated histone demethylase and, until now, no other post-translational modifications have been reported to occur on these enzymes. Therefore, our results show for the first time that both yeast Jhd2 and human JARID1C histone H3 K4 demethylases can be post-translationally modified by ubiquitin in vitro. More importantly, we show that this modification is physiologically relevant and functionally important for maintaining proper histone methylation and gene expression levels.

Recently, it has been shown that LSD1, a non-JmjC domain histone demethylase, needs to associate with CoREST, a known transcriptional corepressor complex, in order to demethylate nucleosomal substrates and to prevent LSD1 degradation by the proteasome (Shi et al. 2005). However, this study did not assess if inhibition of the proteasome could affect LSD1's activity or determine if LSD1's protein level was regulated by the proteasome in an ubiquitin-dependent or -independent manner. Given our results showing that Jhd2 protein level and demethylase activity is controlled by the proteasome in an ubiquitin-dependent manner, it is likely that LSD1 and other mammalian histone demethylases are regulated in a similar manner.

Interestingly, the human homolog of Jhd2, JARID1D, can directly associate with Ring6a/MBLR, a putative RING finger domain-containing ubiquitin ligase (Lee et al. 2007). This direct association was shown to moderately regulate JARID1D demethylase activity in vitro independent of any E1 and E2 activities (Lee et al. 2007). Intriguingly, this study did not determine if Ring6a could ubiquitinate itself, JARID1D, or any other substrates; thus, the substrates for Ring6a are currently unknown. Future studies will be needed to determine 
if human Ring6a or other E3 ubiquitin ligases regulate histone demethylase activity by ubiquitination and to determine if this is a conserved and widely used mechanism.

\section{The role of ubiquitin-mediating enzymes and the} proteasome in controlling histone methylation

Several groups have shown that E2 ubiquitin conjugating enzyme Rad6 and its E3 ubiquitin ligase Bre1 mediate in vivo histone $\mathrm{H} 3 \mathrm{~K} 4$ and $\mathrm{K} 79$ di- and trimethylation via a trans-histone pathway (Latham and Dent 2007; Fingerman et al. 2008). Recently, two independent groups have determined that the E3 ubiquitin ligase Not4 is important for mediating wild-type levels of H3 K4 trimethylation but not H3 K79 methylation (Laribee et al. 2007; Mulder et al. 2007a). While all groups show that Not4 impacts histone $\mathrm{H} 3 \mathrm{~K} 4$ trimethylation, the mechanisms by which Not4 influences histone H3 K4 trimethylation are contradictory (Laribee et al. 2007; Mulder et al. 2007a). For example, it has been reported that a not $4 \Delta$ strain has decreased $\mathrm{H} 2 \mathrm{~B}$ ubiquitination, resulting in reduced PAF transcriptional elongation complex at open reading frames (Mulder et al. 2007a). In contrast, it has also been reported that a not $4 \Delta$ strain has increased levels of H2B ubiquitination and does not affect recruitment of the PAF elongation complex to genes (Laribee et al. 2007). Although this latter group did not determine the mechanism by which Not4 mediates H3 K4 trimethylation, they did show that the 26S proteasome ATPase subunits Cim3 and Cim5 are important in mediating H3 K4 trimethylation (Laribee et al. 2007). It is unclear how to reconcile these contradictory findings. However, our study strongly indicates that the E3 ubiquitin ligase activity of Not4 mediates H3 K4 trimethylation by a polyubiquitin-mediated degradation mechanism that is needed to control the amount of Jhd2 protein and its associated demethylase activity. In addition, our results implicating the proteasome in controlling Jhd2 protein and H3 K4 trimethylation levels could explain why strains expressing temperature-sensitive proteasome ATPase mutants exhibit decreased levels of H3 K4 trimethylation (Ezhkova and Tansey 2004; Laribee et al. 2007).

Until now, Egd2, a protein subunit found in the nascent-associated polypeptide (NAC-EGD) complex, has been the only known substrate for Not4-mediated ubiquitination (Panasenko et al. 2006). In this study, ubiquitination of the NAC-EGD complex, consisting of Egd1 and Egd2, was reduced in a not4 $\Delta$ strain, and in vitro ubiquitination assays showed that Not4 together with Ubc4 is capable of monoubiquitinating Egd2 in the presence of Egd1. Interestingly, in the absence of Not4, the NAC-EGD complex was mislocalized, but the protein stability of Egd1 or Egd2 was not affected (Panasenko et al. 2006). In contrast, our study has identified Jhd 2 as a novel substrate of Not4 that can be polyubiquitinated by Not4 both in vitro and in vivo and that Jhd2 polyubiquitination leads to protein degradation. Therefore, Not4-mediated ubiquitination may have two distinct roles, one for cellular localization and the other for controlling protein levels.

\section{Controlling the balance between histone demethylase and methyltransferase activities}

Although a dynamic balance between histone demethylase and methyltransferase activities must exist for proper histone methylation and gene expression, how this balance is maintained and controlled has not been explored. In our study, we identify a new mechanism that utilizes the E3 ubiquitin ligase activity of Not4 to monitor and control the balance between histone demethylase and methyltransferase activity (Fig. 7E). Because of this control mechanism, Jhd2 levels are kept at extremely low levels, likely explaining why deletion of Jhd2 does not result in a detectable global increase in $\mathrm{H} 3$ K4 trimethylation or gene expression. Therefore, under normal cellular growth conditions, the balance favors maximal H3 $\mathrm{K} 4$ trimethylation at constitutively active genes until monitoring by Not4 is removed.

Interestingly, when yeast are grown in galactosecontaining media, an increase in $\mathrm{H} 3 \mathrm{~K} 4$ trimethylation and gene expression is observed in a $j h d 2 \Delta$ strain at the inducible GAL1 gene (Ingvarsdottir et al. 2007). In addition, Jhd2 is needed to decrease H3 K4 trimethylation levels during the repression of GAL1 (Ingvarsdottir et al. 2007). Therefore, Not4 may also be regulated by environmental cues so that histone methylation can be dynamically altered at inducible and/or constitutive genes. It will be interesting to determine if this is the case and if controlling histone methylation by E3 ubiquitin ligases is a widely conserved mechanism in yeast and higher eukaryotes.

\section{Materials and methods}

\section{Yeast strains and plasmids}

See Supplemental Tables S1 and S2 and the Supplemental Material for details on plasmids and yeast strains.

\section{Yeast extraction and Western blotting analysis}

Yeast extraction and Western blot analysis to detect modified histones was performed as described previously (Briggs et al. 2001; Fingerman et al. 2005). See the Supplemental Material for histone antibodies used. To detect Flag-tagged Jhd2, lysates were run on $8 \%$ SDS-PAGE gels, transferred to PVDF, and immunoblotted with $\alpha$-Flag (Sigma, F7425) used at a 1:5000 dilution. To detect HA-tagged Not4, lysates were run on $8 \%$ SDS-PAGE gels, transferred to PVDF, and immunoblotted with $\alpha$-HA.11 (Covance, MMS-101R) used at a 1:5000 dilution. See the Supplemental Material for details on immunoprecipitation of Jhd2 and Set1 from whole-cell extracts.

\section{In vitro ubiquitination assay}

One microgram of purified CBP-Jhd2 (yeast) or CBP-JARID1C ${ }_{1-700}$ was incubated with $0.5 \mu \mathrm{g}$ of HIS-Not4 (yeast E3) or HIS-hNot4 (human E3), $0.25 \mu \mathrm{g}$ of HIS-Ubc4 (yeast E2) (see the Supplemental Material for expression and purification) or $\mathrm{UbcH} 5 \mathrm{a}$ (human E2) (Boston Biochem), $0.125 \mu \mathrm{g}$ of human Ube1 (E1) 
(Boston Biochem), and $0.75 \mu \mathrm{g}$ of bovine ubiquitin (Sigma) in a reaction mix containing $50 \mathrm{mM}$ Tris $(\mathrm{pH} 7.5), 50 \mathrm{mM} \mathrm{KCl}$, $2.5 \mathrm{mM} \mathrm{MgCl}, 0.5 \mathrm{mM}$ EDTA, $2.5 \mathrm{mM} \mathrm{DTT}$, and $10 \mathrm{mM}$ ATP for various times at $30^{\circ} \mathrm{C}$ as indicated. To inhibit the formation of polyubiquitin chains, $2 \mathrm{mM}$ NEM was added (Panasenko et al. 2006). Samples were separated by SDS-PAGE, transferred to PVDF membrane, and immunoblotted with an $\alpha$-CBP antibody (Millipore, 06-297)

\section{Protein analysis and proteasome inhibition}

Ten-milliliter cultures of cells expressing Flag-tagged Jhd2 in a $\Delta j h d 2 \Delta p d r 5$ strain were treated with DMSO or $0.1 \mathrm{mM}$ MG132 for $30 \mathrm{~min}$. Whole-cell lysates were prepared as described previously (Briggs et al. 2001; Fingerman et al. 2005). Jhd2-Flag and histone $\mathrm{H} 3$ were detected using an $\alpha$-Flag antibody and an $\alpha-\mathrm{H} 3$ antibody. To sensitize yeast to MG132 in a Pdr5-positive strain, yeast were treated as described previously (Liu et al. 2007).

\section{Gene expression analysis}

All quantitative real-time PCR reactions were performed using a StepOne Real-Time PCR System (Applied Biosystems). See the Supplemental Material for details and primer sequences.

\section{ChIP}

ChIPs were performed as described previously using H3 K4 diand trimethyl (Millipore) and histone $\mathrm{H} 3$ antibodies (Abcam) (Kuo and Allis 1999). Immunoprecipitated DNA from three biological repeats with three technical repeats each was analyzed by quantitative real-time PCR using the TaqMan approach. See the Supplemental Material for details and primer sequences.

\section{Acknowledgments}

We thank Mark Hall (Purdue University) for providing the bacterial construct for HIS-Ubc4 expression and helpful discussions and Mary Bryk (Texas A\&M University) for providing the MBY1282 strain. This work was supported by an NIH grant to S.D.B. (GM74183).

\section{References}

Albert, T.K., Hanzawa, H., Legtenberg, Y.I., J.de Ruwe, M., van den Heuvel, F.A., Collart, M.A., Boelens, R., and Timmers, H.T. 2002. Identification of a ubiquitin-protein ligase subunit within the CCR4-NOT transcription repressor complex. $E M B O J$. 21: 355-364.

Briggs, S.D., Bryk, M., Strahl, B.D., Cheung, W.L., Davie, J.K., Dent, S.Y., Winston, F., and Allis, C.D. 2001. Histone H3 lysine 4 methylation is mediated by Set 1 and required for cell growth and rDNA silencing in Saccharomyces cerevisiae. Genes \& Dev. 15: 3286-3295.

Briggs, S.D., Xiao, T., Sun, Z.W., Caldwell, J.A., Shabanowitz, J., Hunt, D.F., Allis, C.D., and Strahl, B.D. 2002. Gene silencing: Trans-histone regulatory pathway in chromatin. Nature 418: 498.

Bryk, M., Briggs, S.D., Strahl, B.D., Curcio, M.J., Allis, C.D., and Winston, F. 2002. Evidence that Set1, a factor required for methylation of histone $\mathrm{H} 3$, regulates rDNA silencing in $S$. cerevisiae by a Sir2-independent mechanism. Curr. Biol. 12: $165-170$.

Cloos, P.A., Christensen, J., Agger, K., and Helin, K. 2008. Erasing the methyl mark: Histone demethylases at the center of cellular differentiation and disease. Genes \& Dev. 22: $1115-1140$.
Collart, M.A. 2003. Global control of gene expression in yeast by the Ccr4-Not complex. Gene 313: 1-16.

Collart, M.A. and Struhl, K. 1994. NOT1(CDC39), NOT2(CDC36), NOT3, and NOT4 encode a global-negative regulator of transcription that differentially affects TATAelement utilization. Genes \& Dev. 8: 525-537.

Collart, M.A. and Timmers, H.T. 2004. The eukaryotic Ccr4-not complex: A regulatory platform integrating mRNA metabolism with cellular signaling pathways? Prog. Nucleic Acid Res. Mol. Biol. 77: 289-322.

Cui, Y., Ramnarain, D.B., Chiang, Y.C., Ding, L.H., McMahon, J.S., and Denis, C.L. 2008. Genome wide expression analysis of the CCR4-NOT complex indicates that it consists of three modules with the NOT module controlling SAGA-responsive genes. Mol. Genet. Genomics 279: 323-337.

Dehe, P.M. and Geli, V. 2006. The multiple faces of Set1. Biochem. Cell Biol. 84: 536-548.

Denis, C.L., Chiang, Y.C., Cui, Y., and Chen, J. 2001. Genetic evidence supports a role for the yeast CCR4-NOT complex in transcriptional elongation. Genetics 158: 627-634.

Dover, J., Schneider, J., Tawiah-Boateng, M.A., Wood, A., Dean, K., Johnston, M., and Shilatifard, A. 2002. Methylation of histone $\mathrm{H} 3$ by COMPASS requires ubiquitination of histone H2B by Rad6. J. Biol. Chem. 277: 28368-28371.

Ezhkova, E. and Tansey, W.P. 2004. Proteasomal ATPases link ubiquitylation of histone $\mathrm{H} 2 \mathrm{~B}$ to methylation of histone $\mathrm{H} 3$. Mol. Cell 13: 435-442.

Fingerman, I.M., Wu, C.L., Wilson, B.D., and Briggs, S.D. 2005. Global loss of Set1-mediated H3 Lys4 trimethylation is associated with silencing defects in Saccharomyces cerevisiae. J. Biol. Chem. 280: 28761-28765.

Fingerman, I.M., Du, H.N., and Briggs, S.D. 2008. Controlling histone methylation via trans-histone pathways. Epigenetics 3: $237-242$.

Hanzawa, H., J.de Ruwe, M., Albert, T.K., Der Vliet, P.C., Timmers, H.T., and Boelens, R. 2001. The structure of the C4C4 ring finger of human NOT4 reveals features distinct from those of C3HC4 RING fingers. J. Biol. Chem. 276: 10185-10190.

Hwang, W.W., Venkatasubrahmanyam, S., Ianculescu, A.G., Tong, A., Boone, C., and Madhani, H.D. 2003. A conserved RING finger protein required for histone H2B monoubiquitination and cell size control. Mol. Cell 11: 261-266.

Ingvarsdottir, K., Edwards, C., Lee, M.G., Lee, J.S., Schultz, D.C., Shilatifard, A., Shiekhattar, R., and Berger, S.L. 2007. Histone H3 K4 demethylation during activation and attenuation of GAL1 transcription in Saccharomyces cerevisiae. Mol. Cell. Biol. 27: 7856-7864.

Klose, R.J., Gardner, K.E., Liang, G., Erdjument-Bromage, H., Tempst, P., and Zhang, Y. 2007. Demethylation of histone H3K36 and H3K9 by Rph1: A vestige of an H3K9 methylation system in Saccharomyces cerevisiae?. Mol. Cell. Biol. 27: 3951-3961.

Kuo, M.H. and Allis, C.D. 1999. In vivo cross-linking and immunoprecipitation for studying dynamic protein:DNA associations in a chromatin environment. Methods 19: 425-433.

Lan, F., Nottke, A.C., and Shi, Y. 2008. Mechanisms involved in the regulation of histone lysine demethylases. Curr. Opin. Cell Biol. 20: 316-325.

Laribee, R.N., Shibata, Y., Mersman, D.P., Collins, S.R., Kemmeren, P., Roguev, A., Weissman, J.S., Briggs, S.D., Krogan, N.J., and Strahl, B.D. 2007. CCR4/NOT complex associates with the proteasome and regulates histone methylation. Proc. Natl. Acad. Sci. 104: 5836-5841.

Latham, J.A. and Dent, S.Y. 2007. Cross-regulation of histone modifications. Nat. Struct. Mol. Biol. 14: 1017-1024. 
Lee, M.G., Norman, J., Shilatifard, A., and Shiekhattar, R. 2007. Physical and functional association of a trimethyl H3K4 demethylase and Ring6a/MBLR, a polycomb-like protein. Cell 128: 877-887.

Liang, G., Klose, R.J., Gardner, K.E., and Zhang, Y. 2007. Yeast Jhd2p is a histone H3 Lys4 trimethyl demethylase. Nat. Struct. Mol. Biol. 14: 243-245.

Liu, C., Apodaca, J., Davis, L.E., and Rao, H. 2007. Proteasome inhibition in wild-type yeast Saccharomyces cerevisiae cells. Biotechniques 42: 158-162.

Miller, T., Krogan, N.J., Dover, J., Erdjument-Bromage, H., Tempst, P., Johnston, M., Greenblatt, J.F., and Shilatifard, A. 2001. COMPASS: A complex of proteins associated with a trithorax-related SET domain protein. Proc. Natl. Acad. Sci. 98: 12902-12907.

Mulder, K.W., Brenkman, A.B., Inagaki, A., van den Broek, N.J., and Timmers, H.T. 2007a. Regulation of histone H3K4 trimethylation and PAF complex recruitment by the Ccr4-Not complex. Nucleic Acids Res. 35: 2428-2439.

Mulder, K.W., Inagaki, A., Cameroni, E., Mousson, F., Winkler, G.S., De Virgilio, C., Collart, M.A., and Timmers, H.T. 2007b. Modulation of Ubc4p/Ubc5p-mediated stress responses by the RING-finger-dependent ubiquitin-protein ligase Not4p in Saccharomyces cerevisiae. Genetics 176: 181-192.

Ng, H.H., Xu, R.M., Zhang, Y., and Struhl, K. 2002. Ubiquitination of histone H2B by Rad6 is required for efficient Dot1mediated methylation of histone H3 lysine 79. J. Biol. Chem. 277: 34655-34657.

Ng, H.H., Robert, F., Young, R.A., and Struhl, K. 2003. Targeted recruitment of Set1 histone methylase by elongating Pol II provides a localized mark and memory of recent transcriptional activity. Mol. Cell 11: 709-719.

Nislow, C., Ray, E., and Pillus, L. 1997. SET1, a yeast member of the trithorax family, functions in transcriptional silencing and diverse cellular processes. Mol. Biol. Cell 8: 2421-2436.

Panasenko, O., Landrieux, E., Feuermann, M., Finka, A., Paquet, N., and Collart, M.A. 2006. The yeast Ccr4-Not complex controls ubiquitination of the nascent-associated polypeptide (NAC-EGD) complex. J. Biol. Chem. 281: 31389-31398.

Peng, J., Schwartz, D., Elias, J.E., Thoreen, C.C., Cheng, D., Marsischky, G., Roelofs, J., Finley, D., and Gygi, S.P. 2003. A proteomics approach to understanding protein ubiquitination. Nat. Biotechnol. 21: 921-926.

Pickart, C.M. 2001. Mechanisms underlying ubiquitination. Annu. Rev. Biochem. 70: 503-533.

Ravid, T. and Hochstrasser, M. 2007. Autoregulation of an E2 enzyme by ubiquitin-chain assembly on its catalytic residue. Nat. Cell Biol. 9: 422-427.

Robzyk, K., Recht, J., and Osley, M.A. 2000. Rad6-dependent ubiquitination of histone H2B in yeast. Science 287: 501-504.

Roguev, A., Schaft, D., Shevchenko, A., Pijnappel, W.W., Wilm, M., Aasland, R., and Stewart, A.F. 2001. The Saccharomyces cerevisiae Set1 complex includes an Ash2 homologue and methylates histone 3 lysine 4. EMBO J. 20: 7137-7148.

Santos-Rosa, H., Schneider, R., Bannister, A.J., Sherriff, J., Bernstein, B.E., Emre, N.C., Schreiber, S.L., Mellor, J., and Kouzarides, T. 2002. Active genes are tri-methylated at K4 of histone H3. Nature 419: 407-411.

Schneider, R., Bannister, A.J., Myers, F.A., Thorne, A.W., CraneRobinson, C., and Kouzarides, T. 2004. Histone H3 lysine 4 methylation patterns in higher eukaryotic genes. Nat. Cell Biol. 6: 73-77.

Seward, D.J., Cubberley, G., Kim, S., Schonewald, M., Zhang, L., Tripet, B., and Bentley, D.L. 2007. Demethylation of trimethylated histone H3 Lys4 in vivo by JARID1 JmjC proteins. Nat. Struct. Mol. Biol. 14: 240-242.
Shahbazian, M.D., Zhang, K., and Grunstein, M. 2005. Histone H2B ubiquitylation controls processive methylation but not monomethylation by Dot1 and Set1. Mol. Cell 19: 271-277.

Shi, Y. and Whetstine, J.R. 2007. Dynamic regulation of histone lysine methylation by demethylases. Mol. Cell 25: 1-14.

Shi, Y.J., Matson, C., Lan, F., Iwase, S., Baba, T., and Shi, Y. 2005. Regulation of LSD1 histone demethylase activity by its associated factors. Mol. Cell 19: 857-864.

Sun, Z.W. and Allis, C.D. 2002. Ubiquitination of histone H2B regulates $\mathrm{H} 3$ methylation and gene silencing in yeast. Nature 418: 104-108.

Tu, S., Bulloch, E.M., Yang, L., Ren, C., Huang, W.C., Hsu, P.H., Chen, C.H., Liao, C.L., Yu, H.M., Lo, W.S., et al. 2007. Identification of histone demethylases in Saccharomyces cerevisiae. J. Biol. Chem. 282: 14262-14271.

Wang, G.G., Allis, C.D., and Chi, P. 2007. Chromatin remodeling and cancer, Part I: Covalent histone modifications. Trends Mol. Med. 13: 363-372.

Wood, A., Krogan, N.J., Dover, J., Schneider, J., Heidt, J., Boateng, M.A., Dean, K., Golshani, A., Zhang, Y., Greenblatt, J.F., et al. 2003. Bre1, an E3 ubiquitin ligase required for recruitment and substrate selection of Rad6 at a promoter. Mol. Cell 11: 267-274. 


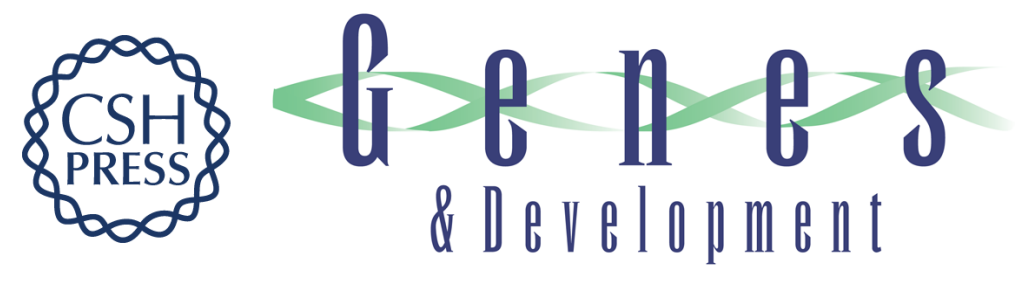

\section{Polyubiquitination of the demethylase Jhd2 controls histone methylation and gene expression}

Douglas P. Mersman, Hai-Ning Du, lan M. Fingerman, et al.

Genes Dev. 2009, 23: originally published online April 3, 2009

Access the most recent version at doi:10.1101/gad.1769209

\section{Supplemental http://genesdev.cshlp.org/content/suppl/2009/04/06/gad.1769209.DC1 Material}

References This article cites 48 articles, 19 of which can be accessed free at: http://genesdev.cshlp.org/content/23/8/951.full.html\#ref-list-1

\section{License}

Email Alerting

Receive free email alerts when new articles cite this article - sign up in the box at the top Service 